\title{
Multilinguales
}

\section{La littérature orale berbère à l'épreuve de l'écriture de Nabile Farès : lecture de la culture en texte}

The berber oral literature put to the test of Nabile Farès writing : reading of culture in text

\section{Farida Boualit et Zahir Sidane}

\section{(2) OpenEdition}

\section{Journals}

Édition électronique

URL : http://journals.openedition.org/multilinguales/1602

DOI : 10.4000/multilinguales. 1602

ISSN : 2335-1853

Éditeur

Université Abderrahmane Mira - Bejaia

Édition imprimée

Date de publication : 1 juin 2014

ISSN : 2335-1535

\section{Référence électronique}

Farida Boualit et Zahir Sidane, «La littérature orale berbère à l'épreuve de l'écriture de Nabile Farès lecture de la culture en texte », Multilinguales [En ligne], 3 | 2014, mis en ligne le 04 juillet 2018, consulté le 17 septembre 2019. URL : http://journals.openedition.org/multilinguales/1602 ; DOI : 10.4000/multilinguales. 1602

Ce document a été généré automatiquement le 17 septembre 2019.

\section{cc) (†)}

Multilinguales est mise à disposition selon les termes de la Licence Creative Commons Attribution -

Pas d'Utilisation Commerciale - Pas de Modification 4.0 International 


\section{La littérature orale berbère à l'épreuve de l'écriture de Nabile Farès : lecture de la culture en texte}

The berber oral literature put to the test of Nabile Farès writing : reading of culture in text

Farida Boualit et Zahir Sidane

1 La production littéraire de Nabile Farès se raréfie ${ }^{3}$. Mais ses textes, surtout ceux des années 70 , continuent de susciter l'intérêt scientifique de la critique universitaire, séduite par l'attrait qu'exerce une écriture qui s'assume comme une expérience des limites dans le sens prôné par Philippe Sollers, dans son ouvrage L'Ecriture et l'expérience des limites (1968):

«Les textes choisis (Dante, Sade, Mallarmé, Lautréamont) se trouvent en position de charnières : d'un côté, ils nous parlent, ils hantent notre discours qui est obligé de leur faire une place en les déformant; de l'autre, et ceci dans leur lettre même, ils sont tournés vers une autre économie que celle qui nous sert à penser l'histoire comme expression, ils restent illisibles. » (p.6)

C'est précisément ainsi que Nabile Farès conçoit explicitement son texte :

«Ce n'est pas parce que nous sommes porteurs de valeurs d'avenir que nous sommes porteurs de valeurs de lisibilité. (...) Moi, je ramène cela à ma propre expérience d'écrivain : je travaille dans la cassure des miroirs, parce que je n'ai pas envie qu'on me rende évident, je n'ai pas besoin de passer ailleurs pour être perçu. Parfois, rendre lisibles les choses, c'est les masquer, c'est créer une reconnaissance où tout le monde étouffe. $»^{4}$

Cette conception du texte-limite ${ }^{5}$ est supportée par un projet d'écriture que cet auteur définit comme la combinaison d'une esthétique et d'une politique, pour paraphraser le narrateur d'un de ses romans, Un passager de l'occident (1971) : «la politique deviendrait poétique. La poétique serait politique » (p.78).

4 Cette « poétique politique » lui est en quelque sorte dictée par son sujet d'écriture : 
"Je suis un maghrébin qui revendique sa berbérité, même si c'est une berbérité encore à construire - elle ne sera jamais ce qu'elle a pu être, si d'ailleurs elle a été à un moment donné du temps. Je suis venu de ce fonds-là, ce qui ne veut pas dire qu'il $\mathrm{y}$ ait refus des autres fonds linguistiques, culturels, qui traversent le Maghreb, mais puisqu'il y a coinçage sur cette réalité, il est important qu'elle soit dite $»^{6}$. le fonds culturel berbère, lui-même à la limite du Sens. En effet, les romans de Nabile Farès sont essentiellement travaillés par la tradition orale berbère du Maghreb. Mais ce n'est pas en cela qu'il est original / marginal/ illisible. De nombreux auteurs maghrébins ont également puisé dans cette tradition des éléments culturels pour alimenter leurs fictions; et de nombreux critiques universitaires ont consacré leurs travaux à cette question, dont l'auteur lui-même.

\section{Le culturème de l'ogresse dans la littérature maghrébine}

6 Au Maghreb, la littérature orale - notamment le conte merveilleux - est fortement présente dans la littérature écrite, qu'elle soit francophone, arabophone ou amazighophone. Camille Lacoste-Dujardin (1975), dans une étude consacrée à la littérature orale populaire maghrébine ${ }^{7}$, justifie ainsi sa pérennité :

«La littérature orale populaire maghrébine peut sans aucun doute figurer au premier rang des littératures orales du monde entier, tant par sa qualité, tout à fait remarquable, que par sa richesse. Qualité qui peut être due, en partie, au fait qu'elle se trouvait encore vivante, encore en fonction, il y a un peu plus d'un siècle, dans des conditions peut-être particulièrement propices à l'épanouissement d'une grande littérature orale; richesse fécondée par la rencontre de deux courants qui s'y sont étroitement mêlés: un vieux fonds méditerranéen d'inspiration très ancienne (...) a été complété par des apports appartenant au même fonds universel (...).» (p. 249) .

7 Comme le souligne l'auteur, la littérature orale maghrébine est riche d'une tradition orale d'origine ancienne mais encore "vivante» dans le temps et les imaginaires collectifs maghrébins. Poésie, contes, légendes, devinettes, proverbes, etc., ponctuent le quotidien des sociétés berbères contemporaines. Nous n'en voulons pour preuve que les nombreux recueils de contes berbères d'ogresse, conçus sur le même modèle que les anciens (quand ceux-ci ne sont pas simplement réactualisés), qui sont encore édités de nos jours au Maghreb et ailleurs (surtout en France), ainsi que sur la toile9.

Camille Lacoste-Dujardin (2010), qui constate dans le même article, que le conte kabyle occupe une place de choix au sein des contes oraux du Maghreb (p.251), souligne ce lien étroit entre le conte kabyle et la vie en société :

«Ainsi les contes recueillis au sein de la Kabylie paysanne semblent-ils profondément enracinés au cœur de cette formation sociale qui apparaît à travers ces récits avec son système de valeurs propres, ses représentations et aussi ses ambiguïtés, ses conflits, ses problèmes. » (p. 252)

9 Ce lien, qui explique à la fois la perpétuelle rémanence du conte kabyle et sa régénérescence, est en quelque sorte confirmé à travers sa convocation permanente par les écrivains maghrébins ${ }^{10}$. Présent dans leurs récits autobiographiques ou autofictionnels, le conte est associé à ce moment de l'enfance dont le rituel contique est partie intégrante, comme dans La Colline oubliée (Plon, 1952) de Mouloud Mammeri : 
«Un soir d'octobre que ma mère, selon son habitude, débitait de sa voix lente et monotone le conte de Hamama de Siouf assise au bord de la mer devant un auditoire émerveillé de garçons et de filles, quelqu'un poussa la porte sans frapper. Il bougonna un bonsoir (...) sans venir se joindre au groupe au coin du feu (...). Comme si de rien n'était, ma mère continua son récit (...). Quand ma mère eut fini, l'étranger sortit de l'ombre et vint droit à elle. » (p. 33) $)^{11}$

Mokrane restitue fidèlement le rituel du conte, mais de l'extérieur de l'«auditoire émerveillé ». Tout en faisant partie du "groupe au coin du feu », comme l'indique le verbe déictique "venir (se joindre)», le jeune homme le décrit à travers un "récit itératif $»^{12}$ qu'il installe dans la «monotonie» de la familiarité. Celle-ci est à situer du côté de l'« habitude » et non de la lassitude, comme en témoigne sa participation au rite, dans le respect de la parole conteuse. Cette attitude de détachement, qui peut s'expliquer par l'âge - Mokrane est sur le point de se marier -, tranche avec celle de " l'intrus » qui, tout en veillant à ne pas perturber le cérémonial, reste à l'écart jusqu'à ce qu'il ait pris fin. Les comportements de la conteuse, qui n'interrompt pas son récit à l'arrivée de «l'étranger ", l'attitude de Mokrane, qui se montre respectueux des règles du rituel, et celle de l'« auditoire » mixte, fasciné par l'histoire, confèrent au rituel social du conte, plus qu'au conte lui-même, une aura sacrée. Dans Le Fils du pauvre de Mouloud Feraoun (Seuil, 1954), le rituel contique est envisagé du côté de l'enfant Fouroulou qui, par adhésion "émerveillée ", semble faire partie de l'«auditoire de garçons et de filles » de La Coline oubliée de Mouloud Mammeri :

«Souvent Nana veille, pour avancer son ouvrage (...). Pendant que Nana travaille nous racontons des histoires.(...) Pendant les récits, nous étions elle (Khalti) et moi, des êtres à part. (...) Je devenais arbitre et soutien du pauvre orphelin qui veut épouser une princesse ; j'assistais tout-puissant au triomphe du petit M'Qidech qui a vaincu l'Ogresse (...). L'histoire coule de la bouche de Khalti et je la bois avidement. » (p.46)

11 Cette adhésion au rituel contique de Fouroulou n'est pas incompatible, comme dans $L a$ Colline oubliée, avec la modalité itérative de ses récits :

«(...) je me souviens bien d'une soirée de printemps ou d'été. Il faisait clair de lune, nous étions dans la courette, Khalti, Baya et moi. Khalti me racontait pour la vingtième fois l'histoire du voleur de paille que le bon dieu voulut confondre (...). L'histoire avait d'ailleurs ses variantes (...). Mais l'idée était la même. » (p.72)

12 Environ deux décennies plus tard, en 1970, le narrateur adolescent,Yahia, dans le premier roman de Nabile Farès, Yahia, pas de chance, évoque la même tradition orale que Mokrane et Fouroulou, toujours en Kabylie, à Akbou, chez l'oncle Saddek et tante Aloula :

«Et oncle Saddek avait continué de parler ce même langage par lequel il aimait traduire des jours en terre de Kabylie et il avait demandé à Yahia " tu te rappelles l'histoire de Mqidech-la-misère et de l'Ogresse aveugle, celle de Mqidech, père de malheur / qui ne dort, / et n'a pas sommeil (...)" ». (pp. 54-55)

13 Nous pourrions multiplier les exemples de la résurgence du conte oral dans la littérature écrite maghrébine contemporaine pour étayer davantage, si besoin était, la thèse selon laquelle le conte participe de l'identité culturelle des sociétés maghrébines. C'est ce qui justifie que nous le qualifiions de "culturème» ou "désignateur de référent culturel ", au sens de Michel Ballard (2003) ${ }^{13}$ :

«Les formes signifiantes sont constituées par des signes de plusieurs sortes (lexèmes, grammèmes, etc.) et les relations qui les unissent. Celles auxquelles nous allons nous intéresser ici sont les désignateurs de référents culturels (DRC), c'est-àdire des signes renvoyant à des éléments ou traits dont l'ensemble constitue une 
civilisation ou une culture. La plupart des DRC sont des signes à désignation explicite, ce sont les culturèmes, qui peuvent être des noms propres (The Wild West) ou des noms communs (porridge) mais il faut être conscient que la désignation peut être effectuée de façon plus implicite, donc plus opaque, à l'aide de pronoms ou de substituts et même de l'ellipse. $»^{14}$

Mais, si le statut de culturème ou DRC du conte kabyle ou berbère au sein des sociétés maghrébines peut être aisément concevable, dans un grand nombre d'œuvres littéraires - celles que nous citons ici et bien d'autres ${ }^{15}-$, le conte fonctionne surtout comme un marqueur identitaire d'une culture menacée.

Dans La Colline oubliée, c'est la mobilisation massive des jeunes de tous les villages, qui partaient en France, pendant la seconde guerre mondiale, qui bouleversait la vie en Kabylie :

«Le mur répercutait sans fin et démesurément les cris de ces femelles à qui l'on enlève leurs petits. (...) Plus personne ne pensait au respect des convenances et dans le deuil à la fois immense et général qui frappait tout Tasga, les femmes de toutes les maisons coudoyaient tous les hommes dans la rue. C'était l'heure où partaient les mobilisés. C'est que tout avait changé depuis que les hommes devaient partir. (...) C'était donc cela la guerre! Tasga ne se remettait pas du mal dont elle souffrait (...).» (p. 31)

L'intrus, qui se tient en retrait du rituel contique, n'est autre qu'Idir dont le lecteur apprend, immédiatement à la fin de la narration du conte, la « mobilisation prochaine » (p. 33).

Dans Le Fils du pauvre, c'est aussi un deuil, ou plutôt un double deuil, qui atteint le rituel : d'abord la mort de Nana, la sœur de la conteuse Khalti, puis la disparition de Khalti elle-même :

"Aucune trace de Khalti, malgré une semaine de recherches. (...) Dire que nous pleurâmes beaucoup Khalti serait, peut-être, exagéré car depuis la mort de Nana, c'était en quelque sorte, le deuil permanent dans la maison (...). Nous, les enfants, nous comprîmes également que nous perdions quelque chose: la maisonnette de ma tante fut vendue par mon père à un voisin qui abattit tout de suite la cloison. » (pp. 87-88)

La «mort» (sans sépulture) de la conteuse et la destruction de l'espace protecteur du rituel du conte marquent la fin de l'enfance et la fin de la première partie de l'œuvre, «La Famille »; la seconde étant intitulée significativement « Le Fils aîné ».

Dans Yahia, pas de chance, le coup d'arrêt porté au rituel est le fait également de la guerre, mais celle des Algériens contre l'occupant français à partir de 1954. La répression provoque à la fois la disparition du conteur (oncle Saddek) et la destruction de l'espace de production de la parole conteuse :

«Et oncle Saddek avait dit, avec une intonation que Yahia ne lui avait pas connue jusqu'à ce jour, et qui marquait, par-delà les complicités et les joies qu'il avait connues lors d'anciennes vacances où il avait apprécié dans l'envoûtement de son âme le joueur de mandoline et le conteur d'histoires, la fin d'un temps de charmes. (...) l'étonnement qui avait gagné Yahia à la vue des deux Jeeps s'était changé en une crainte indicible lorsque, d'un coup poing, le militaire aux cheveux roux avait ouvert la porte de la maison, pris oncle Saddek par le col étroit de sa veste (...). (...) désormais aucune trace d'oncle Saddek ne serait visible au village d'Akbou. Ce même soir tante Aloula et Yahia prenaient le train en gare de Tazmalt. » (pp. 58-59)

20 Ainsi, dans tous ces textes, au-delà des spécificités esthétiques inhérentes à chaque auteur, au-delà de l'écart générationnel, le rituel du conte est considéré comme une composante culturelle d' "un temps de charmes", que les guerres, la mort et les 
disparitions vont détruire en l'atteignant jusque dans son espace. Cependant, alors qu'en général, « la fin des récits » est simplement thématisée comme dans la littérature des années 50 , chez Nabile Farès, l'entreprise est plus radicale ${ }^{16}$.

\section{L'ogresse et Mqidech dans les romans de Nabile Farès}

21 Comme pour le point précédent, de nombreux travaux ont été consacrés à cette question, mais nous allons tenter d'éviter la redondance pour apporter notre modeste pierre à l'édifice.

Ainsi, les culturèmes ou DRC auxquels nous nous intéressons, parce qu'ils sont les plus fréquemment convoqués dans la littérature maghrébine, sont des signes à désignation explicite puisque ce sont: des noms propres (T-s-eryel/ Mqidech-e-), des noms communs («l'ogresse »), et nous ajoutons les signifiants périphrastiques (« celui qui ne dort pas et n'a pas sommeil»), apposés quasi systématiquement au signifiant Mqidech(e) au point de former avec lui une unité par lexicalisation.

Notre choix de ces culturèmes s'explique par l'intérêt que leur porte Nabile Farès. En effet, outre qu'ils sont fortement présents dans ses œuvres de fiction, il leur a également consacré une grande partie de ses travaux de recherche dont une thèse publiée, en 1994, sous le titre L'ogresse dans la littérature orale berbère: littérature orale et anthropologie. Dans celle-ci, l'auteur, en même temps qu'il met au jour leur dimension anthropologique, dévoile quelques clefs de lecture de leur présence dans ses romans.

Nabile Farès confirme, dès le début de son ouvrage, l'importance, au Maghreb, des contes qui associent l'ogresse et Mqidech :

«Le thème du Petit Poucet, c'est-à-dire de la lutte d'un enfant chétif mais rusé, contre l'ogre, ou plutôt ici, l'ogresse dont il finit par venir à bout, est l'un des plus fréquemment traité dans toute la Berbérie. Le Héros est extrêmement populaire : d'un bout à l'autre de l'Afrique du Nord, il porte le nom de Mqidech, Queddidech, Dequidech (...), Haddidouan. » (p. 10)

Ajoutons que, dans la région de Bejaia, il a aussi pour nom Belaâjout (ou Bel Aajout/ Bel Aajouth).

A cette présence significative, il ajoute l'origine obscure du mot « ogresse » : «(...) nous ne savons pas d'où ce terme est venu » (p.. 6) ; affirmation explicitée dans une note en bas de page ${ }^{17}$.

Toujours, au début de l'ouvrage, il souligne également deux caractéristiques de cette ogresse qu'il exploite dans son écriture : sa féminité et sa berbérité absolues :

«Ce terme est celui de tseriel, ou teriel ou tagrog, dont à notre connaissance, l'équivalent masculin n'existe pas. En langue française, ce terme est traduisible (...) par celui d'ogresse ; mais le terme français d'ogresse, féminin du masculin ogre, ne recouvre pas, par exemple, (...) les ensembles sémantiques que ce mot désigne. » (p. 7)

Et enfin, Nabile Farès tient à préciser que l'imaginaire, restreint au merveilleux, est indûment amputé du fantastique, "l'autre pôle de la création et de la structure imaginative » (p.14) :

« De ces deux catégories de l'imaginaire - merveilleux - fantastique -, nous pensons qu'elles ne s'excluent nullement l'une l'autre à l'intérieur d'un récit (...). Tout fantastique n'est donné que sur fond de merveilleux et isoler l'un des termes, c'est 
manquer l'ambiguïté où se situe toute création de l'imaginaire. C'est pourquoi,

l'ogresse peut intervenir comme être bénéfique ou maléfique (...).» (p. 14)

héritière à la fois d'une tradition primitive et d'une autre ogresse, celle d'un texte fondateur de la littérature maghrébine, Nedjma, célèbre personnage éponyme de l'ouvrage de Kateb Yacine (1956), décrite comme «l'ogresse au sang obscur » (p.179), « l'ogresse qui mourut de faim après avoir mangé ses trois frères » (p.180).

37 Tout en exploitant l'origine non recouvrable du vocable «ogresse », Nabile Farès déplace la filiation de la nature (sang obscur) vers la culture (nom obscur) pour signifier que ce qui est irrémédiablement perdu, au Maghreb, depuis le commencement, se situe du côté de la sémantique :

«Maghreb est avant tout un terme de langue : d'une langue. Et une dérive entre deux bords, l'un de l'origine, l'autre de la limite, et, du but (...). Celle-ci (la catégorie Maghreb) a surgi sémantiquement et historiquement "déjà" comme envers, point de départ et d'éloignement de l'origine, d'un Orient. (...) A suivre la catégorie sémantique du Maghreb, on s'aperçoit qu'elle est fondée à partir du sens d'étrangeté" à l'origine. D'où cette superposition existentielle avec son "autre" d'immigré, de Ghareb, d'exilé. » (pp. 209-210) ${ }^{19}$

Ce « Maghreb au nom obscur », dès le commencement, sied à l'Ogresse farésienne d'abord de par l'origine obscure de son nom « ogresse » qui l'habilite à régner sur un territoire 
qui échappe ainsi à la loi monologique de la généalogie parce que " toutes les généalogies remontent à l'Un. Donc : Fausses » (Nabile Farès, 1974 : p. 20) ${ }^{20}$. comme lui,
Chez Nabile Farès, en effet, l'exil est ontologique, car comme l'explique le narrateur de Le Champ des Oliviers: "On a toujours été maintenu entre plusieurs histoires et plusieurs vies. Sans aucune véritable appartenance » (p.23).

Cet «éloignement de l'origine " ouvre la voie au sujet multiple. Ainsi, dans Le Champs des Oliviers, par exemple, le narrateur Brandy Fax est un pseudonyme : " (Brandy Fax est le nom que je me suis donné pour développer (moi le primitif de l'Ancien Monde) un panorama tendu d'occidentalité : limite de deux mondes) »(p.15).

«Brandy Fax (...) a remis bouclé ce qu'il voulait faire sur l'un des êtres les plus fondamentaux que connût l'A.F.N. L'O... Un doute subsiste. Cette thèse sur la signification de l'Ogresse peu commune et peu connue en Europe ne verra pas le jour (la nuit) (le jour) (peut-être pas)? (Qui sait ?) L'obsession? (...) Car je suis un scientifique. Moi. Brandy Fax. (...) L'assistance fut très nombreuse. » (pp. 16-17)

Dans le même chapitre, Brandy Fax/Nabile Farès va s'identifier aux signifiants des deux culturèmes évoqués plus haut, mais légèrement infléchis au niveau de leurs signifiants : Mqidech/Mqides et l'ogresse/l'ogresse :

"Oui. Moi Brandy Fax. Je hais l'Amérique. (...) Parce que depuis que j'ai rencontré l'Amérique. J'ai perdu mon ancien nom. Oui. Le mien. Celui que l'on donne chez nous ( ?) Chez nous? Mqides. Oui. Mqides. Oui. Celui qui ne dort et n'a pas sommeil. Mqides. Père de malheur. Qui ne dort et n'a pas sommeil. Aussi je veille. Je veille. A une dénonciation du monde qui m'a été donné de connaître. (...) Oui. Moi. Mqides. Et il était vingt-trois heures trente lorsque j'entrai directement en contact avec l'être. Cet être. Le plus dévorant du monde. Ce monde

... Que dire de mes anciennes origines? Pas grand-chose. Modernement parlant. Puisque j'appartiens à une origine du monde. Une origine mythique du monde. (...) Chronos. Gargantua. Barbe-Bleue. Hanga-Hanga. Awarzenia...Et bien d'autres. Doivent leur origine à ce premier prodige de l'origine du monde. Ce simple jeu. Entre Moi. Mqides. Et cet être prodigieux Enorme. Mqides. Qui ne dort et n'a pas sommeil. L'Ogresse. Oui. L'Ogresse. Je l'ai eue. Chez moi. En moi. Là. Partout en moi. Dans l'origine prodigieuse Enorme du monde. » (p. 25)

La flexion que subissent les signifiants /Mqidech/ et /ogresse/, dans leur matérialité respective, ([S][s] - ([o] [0]), marque à la fois le lien et la distance de l'écriture farésienne avec le conte en tant que récit dans la tradition orale berbère. En effet, puisque la modalité du «narrer » n'est plus possible, «modernement parlant », l'écriture ne va pas évoquer ou thématiser ces culturèmes.

4 Elle va s'appliquer à mettre au jour leur modalité de signifiance, au sens de Julia Kristeva ${ }^{21}$, et pointée par l'origine non établie du mot « ogresse », l'absolue féminité de l'ogresse berbère, et surtout son ambivalence constitutive entre le fabuleux et le fantastique, telle que définie par N. Farès. Il s'agit de la modalité « sémiotique » de cet " hétérogène au sens et à la signification " (p.232), qu'elle assimile à la chôra, en référence au Timée de Platon dont elle retient qu'elle est un: "réceptacle (...), innommable, invraisemblable, bâtard, antérieur à la nomination, à l'Un, au père, et par conséquent connoté maternel à tel point que "pas même le rang de syllabe" ne lui convient » (p. 233).

Les deux culturèmes, tels que déployés dans l'espace de l'écriture, correspondent tout à fait à cet hétérogène ou chôra. L'Ogresse (avec 0 majuscule la désignant comme signifiant) est placée à l'origine de l'écriture d'un texte-limite qui se veut refuge (fictif) 
des «traces distinctives » de la culture berbère, primitive, archaïque, sur laquelle veille Mqides/Brandy Fax/Nabile Farès. C'est la déduction que l'on peut aisément faire à la lecture des nombreux passages aux allures de manifestes pris en charge par l'Ogresse dans Le Champ des Oliviers :

« ....... L'Ogresse ? Oui... Je suis là... Tout près... Très près... Au-dessous et au-dessus du monde...Je.... Voyage. (...) Longtemps... Bien longtemps... Plusieurs siècles... (...)Trois ou quatre millénaires... (...)...Oui... Je suis là... /... à l'origine des mots... : à l'origine de la formulation des mots... / ... à l'origine de la signification des mots... / ... (...) à l'origine de la création langagière.../...Oui.../...l'origine de la création populaire... /... à l'origine de l'imagination populaire.../...de la sémantique populaire... /... de l'univers des formes populaires... (...) MOI...L'Ogresse de l'origine...Celle qui dévore toutes les interdictions.../... Celle qui exige toutes les permissions.../...Qui condamne la peur... (...) Qui anime l'intelligence... (L'intelligence populaire).../...Qui crée l'intelligence populaire.../Oui. Moi... L'Ogresse d'origine...L'Ogresse aux noms multiples.../...aux jeux multiples.../...aux apparences multiples.../ (...) ... Oui C'est bien Moi/Venue pour Toi/L'Ogresse/ Mqides/Brandy Fax /C'est moi qui/ ». (pp. 27-32)

Dans ses multiples interventions, au style direct, scandées par les points de suspension, les barres obliques d'interruption, les blancs, les phrases incomplètes et inachevées, etc., l'Ogresse est la stratégie d'écriture et la métalangue d'un texte-limite. Sa modalité sémiotique tire sa légitimité de la « culture mineure $»^{22}$ qu'elle veut articuler :

«Moi. L'Ogresse native. Qui attendait la venue de cet homme. Et qui fut trompée par la vertu scripturale de cet homme. Qui. Oui. Ne cessa. Devant moi. Sous moi. D'écrire. Et qui. Ainsi. Me trompa. Trompa l'ogresse native (naïve) que j'étais. Car (...) Et c'est ainsi (dans mon émerveillement) que je fus eue. Par cet homme venu d'une ville. En un jeûne de quarante jours/ quarante nuits d'écriture d'où naquit ce livre qui refoula mon origine vers l'enfance du monde et des hommes. (...) Je dus m'enfuir. Descendre au plus profond des fonds de la terre et du jour. Vieillir. Oui. Vieillir. (..) Mais. Aujourd'hui. (...) Je redécouvre une sorte de jouissance vitale. » (Farès, 1974 : p. 87)

De l'aveu même de l'Ogresse, elle doit son "défouissement " au narrateur du Champs des Oliviers, Brandy Fax, qui tient de la réalité (celle l'auteur) et de la fiction (celle de Mqides) : "Sans lui (Brandy Fax) je continuerais (sans aucun doute) mon sommeil séculaire dans quelque grotte d'Ikharshushen" - "(village perdu au-dessus d'Akbou)»(p. 85). Mais cette culture de l'Ogresse ne sort indemne de long " enfouissement ", comme l'explique dans Le Champ des Oliviers celui que tous les indices désignent comme étant Yahia de Yahia, pas de chance:

"Toutes ces autres années où il nous fallut chercher... O combien chercher... La forme d'une persistance... Apprendre à détourner notre langue... Langue de nos langues... Apprendre à dissimuler nos voyelles...nos syllabes...à les rendre imperceptibles... peu identifiables... à les chuchoter... à les chuinter. (...) Quelquesuns ... (...) ont même été jusqu'à écrire que notre langue était une sorte de bouillie... Incompréhensible... (...) Ils avaient oublié que nous n'avions continué de parler qu'à l'étroit. » (p. 222-223)

C'est donc la nature et le statut de cette culture incarnée par l'Ogresse qui dictent, en quelque sorte, la modalité sémiotique de son écriture qui se préserve de l'hégémonie du sens ses codes.

Nous pouvons dire, au terme de cette investigation non exhaustive, que la figure de l'Ogresse farésienne, est la réactivation d'une Antécédence qui, grâce à sa dualité constitutive, est investie du pouvoir structurant de l'espace du texte; seul espace où 
l'écriture peut impliquer la culture berbère, incarnée en ses culturèmes, dans une stratégie d'oscillation entre sa déterritorialisation nécessaire et la tentation de sa reterritorialisation.

\section{BIBLIOGRAPHIE}

BAKHTINE Mikhail, Esthétique et théorie du roman, Paris, Gallimard, 1978.

BALLARD Michel, Versus : la version réfléchie : anglais-français. vol. 1. Repérages et Paramètres, Gap, Paris, Ophrys, 2003.

BOUALIT Farida, Pour une poétique chromatographique : les cinq textes-programmes de Nabile Farès ", thèse de doctorat soutenue à Paris 8, sous la direction de Claude Duchet, en 1993.

BOUALIT Farida, «L'ogresse farésienne : de l'oralité du conte à l'oralité de l'écriture ou du fabuleux au sémiotique ", in Revue de la faculté des lettres et des sciences humaines, " Ecriture et oralité », numéro spécial (8), Université Sidi Mohamed Abdellah de Fès, 1992, pp. 31-41.

DELEUZE Gilles et GUATTARI Felix, Pour une littérature mineure, Minuit, 1975. DIB Mohammed, Baba Fekrane, contes pour enfants, La Farandole, 1959.

FARES Nabile, Yahia. Pas de chance, Seuil, 1970.

FARES Nabile, Un Passager de l'Occident, Seuil, 1970.

FARES Nabile, Le Champ des Oliviers, Seuil, 1972.

FARES Nabile, Mémoire de l'Absent, Seuil, 1974.

FARES Nabile, « Valeur bénéfique d'une maghrébinité », in Annuaire de l'Afrique du Nord, XXIII, CNRS, 1984, pp. 210-212.

FARES Nabile, L'ogresse dans la littérature orale berbère, Karthala, 1994.

FARES Nabile et KHELIF Kamel, La petite arabe qui aimait la chaise de Van Gogh, Ed. Amok, 2002.

FERAOUN Mouloud, Le Fils du pauvre, Seuil, 1954.

GENETTE Gérard, Figures III, Seuil, 1972.

GANS-GUINOUNE Anne-Marie, De l'impuissance de l'enfance à la revanche par l'écriture, L'Harmattan, 2005.

KRISTEVA Julia, « Le sujet en procès », in Claude Levi Strauss, L'Identité, Grasset, 1977, pp. 223-256.

LACOSTE-DUJARDIN Camille, Contes de femmes et d'ogresses en Kabylie, Karthala, 2010.

LACOSTE-DUJARDIN Camille, « Littérature orale populaire maghrébine. Le conte en berbère : l'exemple du conte kabyle ", in Annuaire de l'Afrique du Nord, Editions du CNRS, vol. 12, 1974, pp. 249-257.

MAMMERI Mouloud, La Colline oubliée, El Othmania pour les pays du Maghreb, 2007.

MAMMERI Mouloud, Machaho : contes berbères de Kabylie, Bordas, 1980. 
TODOROV Tzvetan, Le principe dialogique. Ecrits du cercle de Prague, Seuil, 1981. SAADI Nourredine, « Nabile Farès », in Littérature maghrébine d'expression française, Edicef/Aupelf, 1996. Disponible sur le site : <www.limag.com/Textes/Manuref/fares.htm>.

\section{NOTES}

1. Selon Mikhaïl Bakhtine, cité par T.Todorov en référence aux textes de l'auteur dans leur version d'origine, « (...) le monologisme nie l'existence en dehors de soi d'une autre conscience, ayant mêmes droits et pouvant répondre sur un pied d'égalité, un autre je égal (tu). Dans l'approche monologique (sous sa forme extrême ou pure), autrui reste entièrement et uniquement objet de la conscience, et ne peut former une conscience autre. On n'attend pas de lui une réponse telle qu'elle puisse tout modifier dans le monde de ma conscience.(...) Le monologue se passe d'autrui, c'est pourquoi, dans une certaine mesure, il objective toute la réalité », Le principe dialogique. Ecrits du cercle de Prague, Seuil, 1981, p. 165.

2. Le terme est désormais consacré par la critique, de même que celui de "reterritorialisation ", en référence à Gilles Deleuze et Felix Guattari, Kafka, pour une littérature mineure, Minuit, 1975.

3. Plusieurs années se sont écoulées sans qu'il ait publié d'œuvre de fiction avant celle cosignée avec Kamel Khélif, en 2002, La petite arabe qui aimait la chaise de Van Gogh (ed. Amok). Il faudra attendre 2010 pour son dernier roman, Il était une fois, l'Algérie, aux éditions Achab.

4. Interview publiée dans l'hebdomadaire algérien Algérie-Actualités, $\mathrm{N}^{\circ} 916$, de la semaine du 05 au 11 mai, 1983.

5. Cette dimension de l'œuvre de Nabile Farès a été traitée dans la thèse de doctorat de Boualit Farida, intitulée: "Pour une poétique chromatographique: les cinq textes-programmes de Nabile Farès ", soutenue à Paris 8, sous la direction de Claude Duchet, en 1993.

6. Cité par SAADI Nourredine dans un article intitulé « Nabile Farès », in Littérature maghrébine d'expression française, Edicef/Aupelf, 1996, p. 113. Disponible sur le site: <www.limag.com/ Textes/Manuref/fares.htm>

7. Camille Lacoste-Dujardin, «LITTERATURE ORALE POPULAIRE MAGHREBINE. LE CONTE EN BERBERE : L'EXEMPLE DU CONTE KABYLE », IN Jean-Claude Santucci, Maurice Flory (sous la responsabilité de), Annuaire de l'Afrique du Nord, Paris, Editions du CNRS, vol.12, 1974, pp. 249-257. URL : http://aan.mmsh.univ-aix.fr/Pdf/AAN-1973-12_33.pdf

8. L'article peut être consulté sur le site : http://aan.mmsh.univ-aix.fr/Pdf/AAN-1973-12_33.pdf

9. Citons, par exemple, Najima Thay Thay Rhozali, Au pays des ogres et horreurs, L'Harmattan, 2000 (certains de ses contes sont consultables sur le site : books.google.fr/books ?isbn =2738490506 ; Larbi Rabdi, Loundja fille de l'ogresse et autres contes berbères de Kabylie, Lundja Yellis $n$ tamaza, L'Harmattan, 2003 ; Youcef Allioui, L'Ogresse et l'abeille. Teryel t tzizwit. Contes kabyles - Timucuha, L'Harmattan, 2007; Benredjdal Lounes, Velaajouth (et Teriel). Disponible sur le site: <www.ilmayen.com > Contes et légendes>, 16 avril 2005 ; Nadia Arezki, Loundja fille de Tseriel, conte diffusé le 24/09/2009 puis le 26/05/2011. Disponible sur le site: www.liberte-algerie.com/.../ loundja-fille-de-tseriel-kan-ya-makan-12205

10. Certains de ces écrivains se sont faits traducteurs en français de contes oraux kabyles; citons Mouloud Mammeri, Machaho: contes berbères de Kabylie, Bordas, 1980 ; d'autres se sont faits conteurs eux-mêmes en écrivant des contes, comme Mohammed Dib, Baba Fekrane, contes pour enfants, La Farandole, 1959 ; etc.

11. La pagination adoptée dans tout l'article est celle de l'édition El Othmania pour les pays du Maghreb, 2007.

12. Selon Gérard Genette, le récit itératif est un «type de récit où une seule émission narrative assume ensemble plusieurs occurrences du même événement (c'est-à-dire, encore une fois, plusieurs événements considérés dans leur seule analogie) ", dans Figures III, Seuil, 1972, p. 148. 
13. BALLARD Michel, Versus : la version réfléchie : anglais-français. vol. 1. Repérages et Paramètres, Gap, Paris, Ophrys, 2003. La définition des « culturèmes/DRC » est reprise par l'auteur dans un article intitulé «La lecture des désignateurs de référents culturels». Consultable sur le site: revistas.ulusofona.pt/index.php/babilonia/article/viewFile/1721/1377

14. Idem.

15. L'importance du conte dans l'œuvre de Driss Chraïbi, par exemple, est souvent soulignée par les critiques comme Anne-Marie Gans-Guinoune, dans De l'impuissance de l'enfance à la revanche par l'écriture. Selon elle : "Certains textes de Chraibi sont écrits avec l'intention évidente de nous ramener au temps du conte. Le ton renvoie au "Il était une fois..." ", L'Harmattan, 2005, p. 32.

16. Dans son article «L'ogresse farésienne: de l'oralité du conte à l'oralité de l'écriture ou du fabuleux au sémiotique », Boualit Farida étudie le passage du style parlé de la parole conteuse à l'oralité sémiotique de la parole de l'écriture dans les œuvres de Nabile Farès. In Revue de la faculté des lettres et des sciences humaines, "Ecriture et oralité ", numéro spécial (8), Université Sidi Mohamed Abdellah de Fès, 1992, pp. 31-41.

17. «L'origine du mot est attestée par ces dictionnaires (Littré et Larousse encyclopédique) selon deux voies, l'une qui lie l'origine du mot à une provenance latine, par dérivation d'Orcus, dieu de l'enfant ; l'autre qui lie l'origine du mot à une altération "probable" (Larousse) de hongre: hongrois. (...). Mais " la formation des langues romanes ne se prête pas à cette dérivation" » (p. 7).

18. Le chronotope est défini par M. Bakhtine comme «ce qui se traduit, littéralement, par «tempsespace»: la corrélation essentielle des rapports spatio-temporels, telle qu'elle a été assimilée par la littérature ", dans Mikhaïl Bakhtine, Esthétique et théorie du roman, Paris, Gallimard, 1978, p. 237.

19. Annuaire de l'Afrique du Nord, XXIII, CNRS, 1984.

20. Mémoire de l'Absent, Seuil.

21. « Le sujet en procès », in Claude Levi Strauss, L'Identité, Grasset, 1977, pp. 223-256.

22. «Mineure» de "culture mineure» est à comprendre ici au sens de «mineure" de «littérature mineure " telle que définie par Gilles Deleuze et Felix Guattari : "Une littérature mineure n'est pas celle d'une langue mineure; plutôt celle qu'une minorité fait d'une langue majeure. (...) Les trois caractères de la littérature mineure sont la déterritorialisation de la langue, le branchement de l'individuel sur l'immédiat-politique, l'agencement collectif d'énonciation. Autant dire que "mineur" ne qualifie plus certaines littératures, mais les conditions révolutionnaires de toute littérature au sein de celle qu'on appelle grande (ou établie) ", Kafka, Pour une littérature mineure, op.cit., pp. 29-33. Ce qui est dit ici de la littérature en particulier est aisément transposable à la culture dont elle est partie intégrante.

\section{RÉSUMÉS}

Notre intention a été de revisiter la figure culturelle de l'ogresse dans la littérature maghrébine, en général, et dans les textes de Nabile Farès, en particulier. Nous avons tenté d'abord de montrer son investissement littéraire en tant que culturème de la tradition orale maghrébine. Puis, nous avons analysé son inscription dans le projet d'écriture poético-politique propre à Nabile Farès, en tant qu'elle participe de la subversion du discours monologique ${ }^{1}$. En effet, l'auteur convoque la figure de l'ogresse, accompagnée souvent de celle de son antagoniste Mqidech, aussi petit et rusé qu'elle est énorme et méchante, non pour rendre son texte " lisible », mais, au contraire, pour le « déterritorialiser $»^{2}$. L' Ogresse, «sortie » du conte berbère, participe 
activement à une entreprise de « dés-écriture/réécriture » de l'espace littéraire marqué par une polyphonie généralisée.

Our intention was to revisit the cultural figure of the ogress in the literature from the Maghreb, in general, and in the texts of Nabile Farès, in particular. We tried to show his literary investment as "culturème" of the oral tradition from the Maghreb. Then, we have analyzed his registration in the poetico-political writing project appropriate to Nabile Farès, as it participates to the monological speech subversion. Indeed, the author convenes the figure of the ogress, often accompanied with that of her antagonist Mqidech, so small and cunning, as she is enormous and nasty, not to make the text "readable", but rather to "deterritorize" it. The Ogress "exited" from the Berber tale, is actively involved in this adventure of "un-write/rewrite", marked by a widespread polyphony.

\section{INDEX}

Mots-clés : conte berbère, ogresse, oralité, culturème, Nabile Farès

Keywords : Berber tale, ogress, orality, cultureme, Nabile Farès

\section{AUTEURS}

\section{FARIDA BOUALIT}

Université Abderrahmane Mira - Bejaia Laboratoire LAILEMM

BOUALIT Farida : professeur de l'enseignement supérieur en lettres et langues françaises, à l'Université Abderrahmane Mira - Bejaia ; chef de projet de l'équipe « lectures anthropologiques des textes maghrébins : nouveaux enjeux pour l'ethnocritique »; membre du laboratoire de recherche-formation en langues appliquées et ingénierie des langues en milieu multilingue (LAILEMM) ; responsable de formations doctorales (D/LMD et école doctorale de français pour le doctorat classique) ; membre du réseau algéro-français LaFEF (langue française et expressions francophones) ; titulaire d'une thèse de doctorat sur Nabile Farès, Pour une poétique de la chromatographie : les cinq textes programmes de Nabile Farès, sous la direction de Claude Duchet; auteur de plusieurs articles sur la littérature d'expression française dont :

- L'écriture fantastique africaine francophone : imitation ou création à travers De l'Autre côté du regard de Ken Bugul, in InterFrancophonies, $\mathrm{n}^{\circ}$ 5, 2012.

- L'école française dans Le Fils du pauvre de Mouloud Feraoun : rite de passage ou rite d'institution ?, in Multilinguales, $\mathrm{n}^{\circ} 1,2013$.

- Désir d'utopie et manque à être dans la littérature maghrébine contemporaine », dans Actes du colloque maghrébin Utopia, organisé par le bureau Maghreb de l'AUF, à Rabat (Maroc), en avril 2013. A paraître.

- Pour une étude de la littérature postcoloniale francophone, dans Actes du colloque international Les Francophonies postcoloniales : textes, contextes, à l'université de Delhi (Inde), en novembre 2013. A paraître.

\section{ZAHIR SIDANE}

Université Abderrahmane Mira - Bejaia Laboratoire LAILEMM

SIDANE Zahir : étudiant de l'université Abderrahmane Mira - Bejaia ; titulaire d'un master en sciences des textes littéraires en langue française ; prépare une thèse de doctorat sur L'écriture du 
silence dans quatre textes de Nabile Farès ; L'Etat perdu, La mort de Salah Baye, Le Miroir de Cordoue et Il était une fois l'Algérie. 